\title{
Properties of Segal's Entropy for Quantum Systems
}

\author{
Andrzej Luczak ${ }^{1}$ • Hanna Podsędkowska ${ }^{1}$ (D)
}

Received: 19 November 2016 / Accepted: 4 February 2017 / Published online: 22 February 2017

(C) The Author(s) 2017. This article is published with open access at Springerlink.com

\begin{abstract}
Properties of Segal's entropy for semifinite and finite von Neumann algebras are investigated. In particular, its invariance with respect to a trace-preserving normal *homomorphism is studied, as well as norm-continuity in the trace norm on the set of bounded in the operator norm density matrices.
\end{abstract}

Keywords Entropy $\cdot$ Von Neumann algebra $\cdot$ State

\section{Introduction}

In the paper, we want to address some questions concerning Segal's entropy for normal states, or, more generally, positive operators, in semifinite von Neumann algebras. A particular instance of this entropy is the celebrated von Neumann entropy defined for density matrices in the algebra $\mathbb{B}(\mathcal{H})$ of all bounded linear operators on a Hilbert space. However, in the case of an arbitrary semifinite von Neumann algebra, where instead of the canonical trace we have a normal semifinite faithful trace, substantial differences between these two entropies show up. These differences become still larger when we deal with a finite trace. The problems investigated are as follows: subinvariance and invariance of Segal's entropy with respect to a unital normal positive linear map, and continuity properties of this entropy with respect to the trace norm.

Hanna Podsędkowska

hpodsedk@math.uni.lodz.pl

Andrzej Łuczak

anluczak@math.uni.lodz.pl

1 Faculty of Mathematics and Computer Science, Łódź University, ul. S. Banacha 22, 90-238, Łódź, Poland 


\section{Preliminaries and Notation}

Let $\mathcal{M}$ be a semifinite von Neumann algebra of operators acting on a Hilbert spaced $\mathcal{H}$ with a normal semifinite faithful trace $\tau$, identity $\mathbb{1}$, and predual $\mathcal{M}_{*}$. By $\mathcal{M}^{+}$we shall denote the set of positive operators in $\mathcal{M}$, and by $\mathcal{M}_{*}^{+}$- the set of positive functionals in $\mathcal{M}_{*}$. These functionals will be sometimes referred to as (non-normalised) states. The set of normalised states, i.e. the elements $\rho \in \mathcal{M}_{*}^{+}$such that $\rho(\mathbb{1})=\|\rho\|=1$ will be denoted by $\mathfrak{S .}$

The algebra of measurable operators $\widetilde{\mathcal{M}}$ is defined as a topological *-algebra of densely defined closed operators on $\mathcal{H}$ affiliated with $\mathcal{M}$ with strong addition + and strong multiplication $\cdot$, i.e.

$$
a+b=\overline{a+b}, \quad a \cdot b=\overline{a b}, \quad a, b \in \widetilde{\mathcal{M}},
$$

where $\overline{a+b}$ and $\overline{a b}$ are the closures of the corresponding operators defined by addition and composition respectively on the natural domains given by the intersections of the domains of the $a$ and $b$ and of the range of $b$ and the domain of $a$ respectively. The translationinvariant measure topology is defined by a fundamental system of neighbourhoods of 0 , $\{N(\varepsilon, \delta): \varepsilon, \delta>0\}$, given by

$$
\begin{aligned}
& N(\varepsilon, \delta)=\{a \in \widetilde{\mathcal{M}}: \quad \text { there exists a projection } p \text { in } \mathcal{M} \text { such that } \\
& \qquad a p \in \mathcal{M}, \quad\|a p\| \leqslant \varepsilon \quad \text { and } \quad \tau(\mathbb{1}-p) \leqslant \delta\} .
\end{aligned}
$$

Thus for operators $a_{n}, a \in \widetilde{\mathcal{M}}$, the sequence $\left(a_{n}\right)$ converges to a in measure if for any $\varepsilon, \delta>0$ there exists $n_{0}$ such that for each $n \geqslant n_{0}$ there exists a projection $p \in \mathcal{M}$ such that

$$
\tau(\mathbb{1}-p) \leqslant \delta, \quad\left(a_{n}-a\right) p \in \mathcal{M}, \quad \text { and } \quad\left\|\left(a_{n}-a\right) p\right\| \leqslant \varepsilon .
$$

The following "technical" form of convergence in measure proved in [12, Proposition 2.7] is useful. Let

$$
\left|a_{n}-a\right|=\int_{0}^{\infty} \lambda e_{n}(d \lambda)
$$

be the spectral decomposition of $\left|a_{n}-a\right|$ with spectral measure $e_{n}$ taking values in $\mathcal{M}$ since $a_{n}-a$, and thus $\left|a_{n}-a\right|$, are affiliated with $\mathcal{M}$. Then $a_{n} \rightarrow a$ in measure if and only if for each $\varepsilon>0$

$$
\tau\left(e_{n}([\varepsilon, \infty])\right) \rightarrow 0 .
$$

For each $\rho \in \mathcal{M}_{*}$, there is a measurable operator $h$ such that

$$
\rho(x)=\tau(x h)=\tau(h x), \quad x \in \mathcal{M} .
$$

The space of all such operators is denoted by $L^{1}(\mathcal{M}, \tau)$, and the correspondence above is one-to-one and isometric, where the norm on $L^{1}(\mathcal{M}, \tau)$, denoted by $\|\cdot\|_{1}$, is defined as

$$
\|h\|_{1}=\tau(|h|), \quad h \in L^{1}(\mathcal{M}, \tau) .
$$

The space of all measurable operators $h$ such that $\tau\left(|h|^{p}\right)<+\infty, p \geqslant 1$, constitutes a Banach space $L^{p}(\mathcal{M}, \tau)$ with the norm

$$
\|h\|_{p}=\tau\left(|h|^{p}\right)^{\frac{1}{p}}
$$

(In the theory of noncommutative $L^{p}$-spaces for semifinite von Neumann algebras, it it shown that $\tau$ can be extended to the $h$ 's as above; see e.g. [4, 10, 12] for a detailed account of this theory.) Moreover, to hermitian functionals in $\mathcal{M}_{*}$ correspond selfadjoint operators in $L^{1}(\mathcal{M}, \tau)$, and to states in $\mathcal{M}_{*}$ - positive operators in $L^{1}(\mathcal{M}, \tau)$. For a state $\rho$ the 
corresponding element in $L^{1}(\mathcal{M}, \tau)$ will be denoted by $h_{\rho}$ and called the density matrix of $\rho$, thus

$$
\rho(x)=\tau\left(x h_{\rho}\right)=\tau\left(h_{\rho} x\right), \quad x \in \mathcal{M} .
$$

In particular,

$$
\tau\left(h_{\rho}\right)=\rho(\mathbb{1}),
$$

so for normalised states, we have for their density matrices the equality $\tau\left(h_{\rho}\right)=1$.

Observe that for a finite (normalised) $\tau$, we have $\mathcal{M} \subset L^{1}(\mathcal{M}, \tau)$ while this is not the case for $\tau$ infinite since then $\tau(\mathbb{1})=+\infty$.

Below we present some simple facts which can be regarded as part of the folklore of noncommutative probability theory.

For an arbitrary $a \in L^{1}(\mathcal{M}, \tau)$ we have the spectral decomposition

$$
|a|=\int_{0}^{\infty} \lambda e(d \lambda) .
$$

Thus for any $\varepsilon>0$, we get

$$
|a| \geqslant \int_{\varepsilon}^{\infty} \lambda e(d \lambda) \geqslant \int_{\varepsilon}^{\infty} \varepsilon e(d \lambda)=\varepsilon e([\varepsilon, \infty]) .
$$

Consequently, we obtain the Chebyschev inequality (cf. [3, Lemma 1.1])

$$
\tau(e([\varepsilon, \infty])) \leqslant \frac{\tau(|a|)}{\varepsilon}=\frac{\|a\|_{1}}{\varepsilon} .
$$

Taking into account the above-mentioned "technical" form of convergence in measure we have

Lemma 1 If a sequence $\left(a_{n}\right)$ of operators in $L^{1}(\mathcal{M}, \tau)$ converges in $\|\cdot\|_{1}$-norm, then it converges in measure.

For $x \in L^{1}(\mathcal{M}, \tau)$, define a functional $x \tau$ on $\mathcal{M}$ by the formula

$$
(x \tau)(z)=\tau(z x), \quad z \in \mathcal{M} .
$$

The Segal entropy of $\rho$, denoted by $H(\rho)$, is defined as

$$
H(\rho)=\tau\left(h_{\rho} \log h_{\rho}\right),
$$

i.e. for the spectral representation of $h_{\rho}$

$$
h_{\rho}=\int_{0}^{\infty} \lambda e(d \lambda),
$$

we have

$$
H(\rho)=\int_{0}^{\infty} \lambda \log \lambda \tau(e(d \lambda))
$$

Following Segal [8], we shall be interested only in the case when $h_{\rho} \in \mathcal{M}^{+}$.

Accordingly, we define Segal's entropy for $h \in \mathcal{M}^{+}$by the formula

$$
H(h)=\tau(h \log h)=\int_{0}^{\infty} \lambda \log \lambda \tau(e(d \lambda)),
$$

where $h$ has the spectral representation as in (1). Let us note that the existence of Segal's entropy is by no means guaranteed, however, for finite $\tau$ and normalised state $\rho$, we have, on account of the inequality

$$
\lambda \log \lambda \geqslant \lambda-1
$$


the relation

$$
\begin{aligned}
H(\rho) & =\int_{0}^{\infty} \lambda \log \lambda \tau(e(d \lambda)) \geqslant \int_{0}^{\infty}(\lambda-1) \tau(e(d \lambda)) \\
& =\tau\left(\int_{0}^{\infty} \lambda e(d \lambda)\right)-\tau\left(\int_{0}^{\infty} e(d \lambda)\right)=\tau\left(h_{\rho}\right)-\tau(\mathbb{1})=0,
\end{aligned}
$$

showing that, at least in this case, Segal's entropy is well defined and nonnegative.

Remark 1 It should be noted that the original Segal definition of entropy differs from ours by a minus sign before the trace. However, for the sake of having nonnegative entropy for states on a finite von Neumann algebra we have adopted the definition as above.

\section{Subinvariance and Invariance of Segal's Entropy}

Let $\alpha: \mathcal{M} \rightarrow \mathcal{M}$ be a normal positive unital linear map such that

$$
\tau \circ \alpha=\tau .
$$

The (pre-)dual map $\alpha_{*}$ is defined on $\mathcal{M}_{*}$ by the formula

$$
\alpha_{*}(\rho)=\rho \circ \alpha, \quad \rho \in \mathcal{M}_{*} .
$$

Our aim is to establish the form of the density matrix of $\alpha_{*}(\rho)$. To this end the following construction is employed.

Fix an element $z \in \mathcal{M}^{h}$, and consider a linear map

$$
f:\left\{x \tau: x \in \mathcal{M}^{h} \cap L^{1}(\mathcal{M}, \tau)\right\} \rightarrow \mathbb{R}
$$

defined by

$$
f(x \tau)=\tau(\alpha(x) z) .
$$

(Since $\overline{\tau(\alpha(x) z)}=\tau\left((\alpha(x) z)^{*}\right)=\tau(z \alpha(x))=\tau(\alpha(x) z)$ the values of $f$ lie in $\mathbb{R}$.) The function $\mathbb{R} \ni t \mapsto|t|$ is convex, thus the Jensen inequality for positive unital maps yields

$$
|\alpha(x)| \leqslant \alpha(|x|),
$$

for each $x \in \mathcal{M}^{h}$. Consequently, we get from the properties of trace

$$
\begin{aligned}
|f(x \tau)| & =|\tau(\alpha(x) z)| \leqslant\|z\|_{\infty} \tau(|\alpha(x)|) \leqslant\|z\|_{\infty} \tau(\alpha(|x|)) \\
& =\|z\|_{\infty} \tau(|x|)=\|z\|_{\infty}\|x\|_{1}=\|z\|_{\infty}\|x \tau\|,
\end{aligned}
$$

which means that $f$ is bounded and $\|f\| \leqslant\|z\|_{\infty}$. Hence $f$ can be extended to a bounded linear functional on $\overline{\left\{x \tau: x \in \mathcal{M}^{h} \cap L^{1}(\mathcal{M}, \tau)\right\}}=\mathcal{M}_{*}^{h}$. This, in turn, yields that $f \in$ $\left(\mathcal{M}_{*}^{h}\right)^{*} \simeq \mathcal{M}^{h}$. Denote the element in $\mathcal{M}^{h}$ corresponding to $f$ by $\widetilde{\alpha}(z)$. We then have

$$
\tau(\alpha(x) z)=f(x \tau)=(x \tau)(\widetilde{\alpha}(z))=\tau(\widetilde{\alpha}(z) x)=\tau(x \widetilde{\alpha}(z)) .
$$

We have thus obtained a map $\mathcal{M}^{h} \ni z \mapsto \widetilde{\alpha}(z) \in \mathcal{M}^{h}$ which is clearly linear. For $\widetilde{\alpha}(z)$ regarded as a linear functional on $\mathcal{M}_{*}^{h}$, we have on account of relation (2)

$$
|\widetilde{\alpha}(z)(x \tau)|=|f(x \tau)| \leqslant\|z\|_{\infty}\|x \tau\|,
$$

which means that

$$
\|\widetilde{\alpha}(z)\|_{\infty} \leqslant\|z\|_{\infty}
$$

so $\|\tilde{\alpha}\| \leqslant 1$. Further, for each $x \in \mathcal{M}^{h} \cap L^{1}(\mathcal{M}, \tau)$, we have

$$
(x \tau)(\widetilde{\alpha}(\mathbb{1}))=\tau(\widetilde{\alpha}(\mathbb{1}) x)=\tau(\alpha(x))=\tau(x)=(x \tau)(\mathbb{1}),
$$


hence

$$
\widetilde{\alpha}(\mathbb{1})=\mathbb{1}
$$

Relations (3) and (4) yield

$$
\|\widetilde{\alpha}\|=1=\|\widetilde{\alpha}(\mathbb{1})\|,
$$

which by virtue of [1, Corollary 3.2.6] shows the positivity of $\widetilde{\alpha}$ (the reasoning in [1, Corollary 3.2.6] is performed for a map between $C^{*}$-algebras while in our case we deal with a map from the hermitian part of $\mathcal{M}$ into itself but the proof of positivity remains exactly the same also in this restricted situation). Now $\widetilde{\alpha}$, being a bounded map, can be extended to a bounded linear map on the whole of $\mathcal{M}$, denoted by the same symbol, and this extended map is obviously positive and satisfies relations (4) and (5). Moreover, since each element in $\mathcal{M}$ is a linear combination of two elements from $\mathcal{M}^{h}$, we have

$$
\tau(\alpha(x) y)=\tau(x \widetilde{\alpha}(y)), \quad x, y \in \mathcal{M} .
$$

For the dual map $\widetilde{\alpha}^{*}$ defined on $\mathcal{M}^{*}$ by the formula

$$
\widetilde{\alpha}^{*}(\varphi)=\varphi \circ \widetilde{\alpha}, \quad \varphi \in \mathcal{M}^{*},
$$

we have, for the functionals $x \tau$ with $x \in \mathcal{M} \cap L^{1}(\mathcal{M}, \tau)$,

$$
\widetilde{\alpha}^{*}((x \tau))(y)=(x \tau)(\widetilde{\alpha}(y))=\tau(\widetilde{\alpha}(y) x)=\tau(y \alpha(x))=(\alpha(x) \tau)(y) .
$$

Since $|\tau(\alpha(x))|=|\tau(x)|<+\infty$, it follows that $\alpha(x) \in \mathcal{M} \cap L^{1}(\mathcal{M}, \tau)$, consequently, $(x \tau) \circ \widetilde{\alpha}=\alpha(x) \tau \in \mathcal{M}_{*}$. This means that

$$
\tilde{\alpha}^{*}\left(\left\{x \tau: x \in \mathcal{M} \cap L^{1}(\mathcal{M}, \tau)\right\}\right) \subset \mathcal{M}_{*},
$$

and since $\left\{x \tau: x \in \mathcal{M} \cap L^{1}(\mathcal{M}, \tau)\right\}$ is dense in $\mathcal{M}_{*}$, and $\left\|\widetilde{\alpha}^{*}\right\|=1$, the relation above yields

$$
\tilde{\alpha}^{*}\left(\mathcal{M}_{*}\right) \subset \mathcal{M}_{*}
$$

showing that $\tilde{\alpha}$ is normal.

Now take arbitrary $z \in \mathcal{M}^{+}$, and let $x_{i} \in \mathcal{M}^{+} \cap L^{1}(\mathcal{M}, \tau)$ be such that $x_{i} \uparrow \mathbb{1}$. Then

$$
0 \leqslant \widetilde{\alpha}(z)^{1 / 2} x_{i} \widetilde{\alpha}(z)^{1 / 2} \uparrow \widetilde{\alpha}(z),
$$

which implies, on account of the normality of $\tau$,

$$
\tau\left(\widetilde{\alpha}(z)^{1 / 2} x_{i} \widetilde{\alpha}(z)^{1 / 2}\right) \uparrow \tau(\widetilde{\alpha}(z)) .
$$

On the other hand, we have, since $\alpha\left(x_{i}\right) \uparrow \alpha(\mathbb{1})=\mathbb{1}$,

$$
\begin{aligned}
\tau\left(\widetilde{\alpha}(z)^{1 / 2} x_{i} \widetilde{\alpha}(z)^{1 / 2}\right) & =\tau\left(x_{i} \widetilde{\alpha}(z)\right) \\
& =\tau\left(\alpha\left(x_{i}\right) z\right)=\tau\left(z^{1 / 2} \alpha\left(x_{i}\right) z^{1 / 2}\right) \uparrow \tau(z),
\end{aligned}
$$

showing that $\tau(\widetilde{\alpha}(z))=\tau(z)$, and thus $\tau \circ \widetilde{\alpha}=\tau$.

The results of our considerations may by summarised as follows. Let $\mathcal{M}$ be a von Neumann algebra with a normal faithful semifinite trace $\tau$, and let $\alpha$ be a normal positive unital linear map on $\mathcal{M}$ such that $\tau \circ \alpha=\tau$. Then there is a normal positive unital linear map $\widetilde{\alpha}$ on $\mathcal{M}$ such that $\tau \circ \tilde{\alpha}=\tau$, and

$$
\tau(\alpha(x) y)=\tau(x \widetilde{\alpha}(y)), \quad x, y \in \mathcal{M} .
$$

The map $\widetilde{\alpha}$ will be called conjugate to $\alpha$.

Lemma 2 Let $\rho \in \mathcal{M}_{*}$ be such that $h_{\rho} \in \mathcal{M}$. Then

$$
h_{\alpha_{*}(\rho)}=\widetilde{\alpha}\left(h_{\rho}\right) .
$$


Proof For each $x \in \mathcal{M}$, we have

$$
\tau\left(x h_{\alpha_{*}(\rho)}\right)=\alpha_{*}(\rho)(x)=\rho(\alpha(x))=\tau\left(h_{\rho} \alpha(x)\right)=\tau\left(\widetilde{\alpha}\left(h_{\rho}\right) x\right),
$$

and the conclusion follows.

Remark 2 The same result can be obtained in a stronger form without the assumption $h_{\rho} \in \mathcal{M}$. However, this would require extension of $\widetilde{\alpha}$ from $\mathcal{M}$ to $L^{1}(\mathcal{M}, \tau)$, which is not needed for our present purposes.

Theorem 3 Let $\mathcal{M}, \tau$ and $\alpha$ be as before. Then for every $\rho \in \mathcal{M}_{*}^{+}$, the following relation holds

$$
H\left(\alpha_{*}(\rho)\right) \leqslant H(\rho) .
$$

Proof Taking into account Lemma 2, we obtain

$$
H\left(\alpha_{*}(\rho)\right)=\tau\left(h_{\alpha_{*}(\rho)} \log h_{\alpha_{*}(\rho)}\right)=\tau\left(\widetilde{\alpha}\left(h_{\rho}\right) \log \widetilde{\alpha}\left(h_{\rho}\right)\right) .
$$

The function $[0,+\infty) \ni t \mapsto t \log t$ is operator convex, and the map $\widetilde{\alpha}$ is positive and unital, so from Jensen's inequality we get

$$
\widetilde{\alpha}\left(h_{\rho} \log h_{\rho}\right) \geqslant \widetilde{\alpha}\left(h_{\rho}\right) \log \widetilde{\alpha}\left(h_{\rho}\right),
$$

consequently,

$$
\tau\left(\widetilde{\alpha}\left(h_{\rho}\right) \log \widetilde{\alpha}\left(h_{\rho}\right)\right) \leqslant \tau\left(\widetilde{\alpha}\left(h_{\rho} \log h_{\rho}\right)\right)=\tau\left(h_{\rho} \log h_{\rho}\right)=H(\rho),
$$

showing the claim.

Remark 3 It should be noted that the result above, in a slightly stronger form, was obtained in [6, Proposition 7.3]. However, the existence of the conjugate map $\widetilde{\alpha}$ satisfying the basic relation (6) was simply taken for granted without showing its properties. Since these properties will be important in our further considerations, we have decided to present in detail the whole construction of this map.

Now we want to investigate the situation where the entropy does not change under a transformation of states.

Theorem 4 Let $\alpha$ be $a *$-homomorphism such that $\tau \circ \alpha=\tau$. Put $\mathcal{N}=\alpha(\mathcal{M})$. The relation

$$
H\left(\alpha_{*}(\rho)\right)=H(\rho)
$$

holds if and only if $h_{\rho} \in \mathcal{N}$.

Proof Let us start with some preliminary analysis. For arbitrary $x, y \in \mathcal{M}$, we have

$$
\tau(\widetilde{\alpha}(\alpha(x)) y)=\tau(\alpha(x) \alpha(y))=\tau(\alpha(x y))=\tau(x y),
$$

which shows that

$$
\widetilde{\alpha}(\alpha(x))=x .
$$

Let $\alpha\left(x_{i}\right) \rightarrow y \sigma$-weakly. Then, as $\tilde{\alpha}$ is normal,

$$
x_{i}=\tilde{\alpha}\left(\alpha\left(x_{i}\right)\right) \rightarrow \widetilde{\alpha}(y),
$$


and the normality of $\alpha$ yields

$$
y=\lim _{i} \alpha\left(x_{i}\right)=\alpha(\widetilde{\alpha}(y)) \in \alpha(\mathcal{M})=\mathcal{N},
$$

which shows that $\mathcal{N}$ is $\sigma$-weakly closed, consequently, $\mathcal{N}$ is a von Neumann algebra. The map $\mathbb{E}=\alpha \circ \widetilde{\alpha}$ is a projection onto $\mathcal{N}$, so it is a conditional expectation, moreover, $\mathbb{E}$ is normal and $\tau \circ \mathbb{E}=\tau$.

For arbitrary $x, y, z \in \mathcal{M}$, we have, since $(\mathbb{E} y) \alpha(z) \in \mathcal{N}$,

$$
\tau(\widetilde{\alpha}(x \mathbb{E} y) z)=\tau(x(\mathbb{E} y) \alpha(z))=\tau(\mathbb{E}(x(\mathbb{E} y) \alpha(z)))=\tau(\mathbb{E} x(\mathbb{E} y) \alpha(z)),
$$

and

$$
\begin{aligned}
\tau(\widetilde{\alpha}(x) \widetilde{\alpha}(y) z) & =\tau(\alpha(\widetilde{\alpha}(x) \widetilde{\alpha}(y) z)) \\
& =\tau(\alpha(\widetilde{\alpha}(x)) \alpha(\widetilde{\alpha}(y)) \alpha(z))=\tau(\mathbb{E} x(\mathbb{E} y) \alpha(z)),
\end{aligned}
$$

which shows that

$$
\widetilde{\alpha}(x \mathbb{E} y)=\widetilde{\alpha}(x) \widetilde{\alpha}(y) .
$$

Since $\tilde{\alpha} \circ \mathbb{E}=\widetilde{\alpha}$, we obtain for arbitrary $x, y \in \mathcal{M}$

$$
\widetilde{\alpha}(\mathbb{E} x \mathbb{E} y)=\widetilde{\alpha}(\mathbb{E} x) \widetilde{\alpha}(y)=\widetilde{\alpha}(x) \widetilde{\alpha}(y),
$$

and consequently,

$$
\widetilde{\alpha}\left((\mathbb{E} x)^{n}\right)=\widetilde{\alpha}(x)^{n} .
$$

It follows that for arbitrary polynomial $W$

$$
\widetilde{\alpha}(W(\mathbb{E} x))=W(\widetilde{\alpha}(x)),
$$

and thus

$$
\widetilde{\alpha}(f(\mathbb{E} x))=f(\widetilde{\alpha}(x))
$$

for an arbitrary function $f$ which is continuous on $s p \widetilde{\alpha}(x) \cup s p \mathbb{E} x$. Observe that since $\alpha$ is a*-homomorphism, we get

$$
\alpha\left(x^{n}\right)=\alpha(x)^{n},
$$

and thus

$$
\alpha(f(x))=f(\alpha(x))
$$

for an arbitrary function $f$ which is continuous on $s p \widetilde{\alpha}(x) \cup \operatorname{spx}$. In particular, for the function $f(t)=t \log t$ we have, for every $x \in \mathcal{M}^{+}$, the equalities

$$
\widetilde{\alpha}(\mathbb{E} x \log \mathbb{E} x)=\tilde{\alpha}(x) \log \tilde{\alpha}(x), \quad \alpha(x \log x)=\alpha(x) \log \alpha(x) .
$$

Assume first that $h_{\rho} \in \mathcal{N}$. Then $h_{\rho}=\alpha(h)$ for some $h \in \mathcal{M}$, and thus $\widetilde{\alpha}\left(h_{\rho}\right)=h$ which shows that $h$ is a density matrix. Taking into account the relations (7) and (8), we obtain

$$
\begin{aligned}
H\left(\alpha_{*}(\rho)\right. & =\tau\left(\widetilde{\alpha}\left(h_{\rho}\right) \log \widetilde{\alpha}\left(h_{\rho}\right)\right)=\tau(h \log h) \\
& =\tau(\alpha(h \log h))=\tau(\alpha(h) \log \alpha(h))=\tau\left(h_{\rho} \log h_{\rho}\right)=H(\rho),
\end{aligned}
$$

Now assume that $H\left(\alpha_{*}(\rho)\right)=H(\rho)$. Since the function $f$ as above is operator convex, and $\tilde{\alpha}$ is positive and unital, it follows from Jensen's inequality that

$$
\mathbb{E} h_{\rho} \log \mathbb{E} h_{\rho} \leqslant \mathbb{E}\left(h_{\rho} \log h_{\rho}\right) .
$$

Consequently, taking into account the relations (8) and (9), we obtain

$$
\begin{aligned}
H\left(\alpha_{*}(\rho)\right) & =\tau\left(\widetilde{\alpha}\left(h_{\rho}\right) \log \widetilde{\alpha}\left(h_{\rho}\right)\right)=\tau\left(\widetilde{\alpha}\left(\mathbb{E} h_{\rho} \log \mathbb{E} h_{\rho}\right)\right) \\
& =\tau\left(\mathbb{E} h_{\rho} \log \mathbb{E} h_{\rho}\right) \leqslant \tau\left(\mathbb{E}\left(h_{\rho} \log h_{\rho}\right)\right) \\
& =\tau\left(h_{\rho} \log h_{\rho}\right)=H(\rho)=H\left(\alpha_{*}(\rho)\right),
\end{aligned}
$$


which shows, in particular, that

$$
\tau\left(\mathbb{E} h_{\rho} \log \mathbb{E} h_{\rho}\right)=\tau\left(\mathbb{E}\left(h_{\rho} \log h_{\rho}\right)\right) .
$$

Consequently, we get

$$
\tau\left(\mathbb{E}\left(h_{\rho} \log h_{\rho}\right)-\mathbb{E} h_{\rho} \log \mathbb{E} h_{\rho}\right)=0,
$$

and the inequality (9) and the faithfulness of $\tau$ yield

$$
\mathbb{E}\left(h_{\rho} \log h_{\rho}\right)-\mathbb{E} h_{\rho} \log \mathbb{E} h_{\rho}=0,
$$

i.e. we have equality in Jensen's inequality. From [5, Appendix B.5], it follows that

$$
\mathbb{E} h_{\rho}=h_{\rho},
$$

which means that $h_{\rho} \in \mathcal{N}$.

As a corollary we obtain

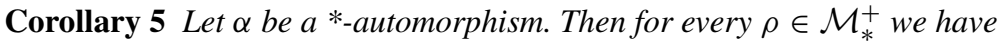

$$
H\left(\alpha_{*}(\rho)\right)=H(\rho) .
$$

\section{Continuity Properties of Segal's Entropy}

In this section we want to investigate the continuity of Segal's entropy.

Lemma 6 Let $\rho \in \mathfrak{S}$, and let $0<\alpha \leqslant \beta$. Then for each $h \in \mathcal{M}^{+}$, we have

$$
\rho\left(h^{\alpha}\right)^{\frac{1}{\alpha}} \leqslant \rho\left(h^{\beta}\right)^{\frac{1}{\beta}} \text {. }
$$

Proof Set $\gamma=\frac{\beta}{\alpha}$. Since $\gamma \geqslant 1$, and the function $\mathbb{R}_{+} \ni t \mapsto t^{\gamma}$ is convex, Jensen's inequality yields

$$
\rho(z)^{\frac{\beta}{\alpha}}=\rho(z)^{\gamma} \leqslant \rho\left(z^{\gamma}\right)=\rho\left(z^{\frac{\beta}{\alpha}}\right),
$$

for each $z \in \mathcal{M}^{+}$. Putting $z=h^{\alpha}$, we obtain

$$
\rho\left(h^{\alpha}\right)^{\frac{\beta}{\alpha}} \leqslant \rho\left(h^{\beta}\right)
$$

which shows the claim.

Define the quantum mechanical Rényi entropy ( $\equiv \alpha$-entropy) $S_{\alpha}, \alpha \neq 1$, by the formula (see e.g. [6, Part II, Chapter 7])

$$
S_{\alpha}(\rho)=\frac{1}{1-\alpha} \log \tau\left(h_{\rho}^{\alpha}\right), \quad \rho \in \mathfrak{S} .
$$

We want to investigate some properties of this entropy.

Proposition 7 Fix $\rho \in \mathfrak{S}$. The function $\alpha \mapsto S_{\alpha}(\rho)$ is decreasing on the interval $(1,+\infty)$.

Proof We have

$$
\begin{aligned}
S_{\alpha}(\rho) & =-\log \tau\left(h_{\rho}^{\alpha}\right)^{\frac{1}{\alpha-1}} \\
& =-\log \tau\left(h_{\rho} h_{\rho}^{\alpha-1}\right)^{\frac{1}{\alpha-1}}=-\log \rho\left(h_{\rho}^{\alpha-1}\right)^{\frac{1}{\alpha-1}},
\end{aligned}
$$


and the conclusion follows from Lemma 6.

Assume that the state $\rho \in \mathfrak{S}$, with the density matrix $h_{\rho} \in \mathcal{M}^{+}$, has finite Segal's entropy, and take a closer look at the function $\alpha \mapsto \tau\left(h_{\rho}^{\alpha}\right)$. Its difference quotient at point 1 equals

$$
\frac{\tau\left(h_{\rho}^{\alpha}\right)-\tau\left(h_{\rho}\right)}{\alpha-1}=\int_{0}^{\infty} \frac{\lambda^{\alpha}-\lambda}{\alpha-1} \tau(e(d \lambda)),
$$

where

$$
h_{\rho}=\int_{0}^{\infty} \lambda e(d \lambda)
$$

is the spectral representation of $h_{\rho}$. For the net of functions $\left\{f_{\alpha}: \alpha>1\right\}$ defined as

$$
f_{\alpha}(\lambda)=\frac{\lambda^{\alpha}-\lambda}{\alpha-1}
$$

a little of calculus shows that for each fixed $\lambda$ we have $f_{\alpha}(\lambda) \leqslant f_{\beta}(\lambda)$, for $\alpha<\beta$, so this net is increasing. Moreover, we have

$$
\lim _{\alpha \rightarrow 1+} f_{\alpha}(\lambda)=\lambda \log \lambda,
$$

and the Lebesgue Monotone Convergence Theorem yields

$$
\lim _{\alpha \rightarrow 1+} \int_{0}^{\infty} f_{\alpha}(\lambda) \tau(e(d \lambda))=\int_{0}^{\infty} \lambda \log \lambda \tau(e(d \lambda))=H(\rho) .
$$

(To be more precise, for passing to the limit we should divide the integral into two parts $\int_{0}^{1}$ and $\int_{1}^{\infty}$, and for the integral $\int_{0}^{1}$ consider the positive functions $\left(-f_{\alpha}\right)$.)

Hence, we have shown that the function $\alpha \mapsto \tau\left(h_{\rho}^{\alpha}\right)$ is differentiable at $\alpha=1$, and

$$
\left[\tau\left(h_{\rho}^{\alpha}\right)\right]_{\alpha=1}^{\prime}=H(\rho) .
$$

Consequently, for the function $\alpha \mapsto \log \tau\left(h_{\rho}^{\alpha}\right)$ we have

$$
\left[\log \tau\left(h_{\rho}^{\alpha}\right)\right]_{\alpha=1}^{\prime}=\frac{H(\rho)}{\tau\left(h_{\rho}\right)}=H(\rho) .
$$

Observe now that for the Rényi entropy we have

$$
-S_{\alpha}(\rho)=\frac{\log \tau\left(h_{\rho}^{\alpha}\right)-\log \tau\left(h_{\rho}\right)}{\alpha-1} \underset{\alpha \rightarrow 1+}{\longrightarrow}\left[\log \tau\left(h_{\rho}^{\alpha}\right)\right]_{\alpha=1}^{\prime}=H(\rho),
$$

consequently, Segal's entropy $H$ is a limit of a decreasing net of functions $\left\{-S_{\alpha}: \alpha>1\right\}$ (note that we consider the limit at 1 , so for $\alpha<\beta$ we have $-S_{\alpha} \leqslant-S_{\beta}$ ).

Now fix $\alpha>1$, and consider the function $\mathfrak{S} \ni \rho \mapsto S_{\alpha}(\rho)$. For $\rho, \varphi \in \mathfrak{S}$ we have the following estimate

$$
\begin{aligned}
\left|\tau\left(h_{\rho}^{\alpha}\right)^{\frac{1}{\alpha}}-\tau\left(h_{\varphi}^{\alpha}\right)^{\frac{1}{\alpha}}\right| & =\left|\left\|h_{\rho}\right\|_{\alpha}-\left\|h_{\varphi}\right\|_{\alpha}\right| \leqslant\left\|h_{\rho}-h_{\varphi}\right\|_{\alpha} \\
& =\tau\left(\left|h_{\rho}-h_{\varphi}\right|^{\alpha}\right)^{\frac{1}{\alpha}}=\left[\tau\left(\left|h_{\rho}-h_{\varphi}\right|\left|h_{\rho}-h_{\varphi}\right|^{\alpha-1}\right)\right]^{\frac{1}{\alpha}} \\
& \leqslant\left[\left\|\left|h_{\rho}-h_{\varphi}\right|^{\alpha-1}\right\|_{\infty} \tau\left(\left|h_{\rho}-h_{\varphi}\right|\right)\right]^{\frac{1}{\alpha}} \\
& =\left(\left\|h_{\rho}-h_{\varphi}\right\|_{\infty}^{\alpha-1}\left\|h_{\rho}-h_{\varphi}\right\|_{1}\right)^{\frac{1}{\alpha}}
\end{aligned}
$$

From this estimate we obtain 
Proposition 8 The Rényi entropy is continuous in the $\|\cdot\|_{1}$-norm on the set $\{\rho \in \mathfrak{S}: \|$ $\left.h_{\rho} \|_{\infty} \leqslant c\right\}$ for every $c \geqslant 0$. Moreover, for $\mathcal{M}=\mathbb{B}(\mathcal{H})$ and the canonical trace $\tau=\operatorname{tr}$, the Rényi entropy is continuous on the whole of $\mathfrak{S}$.

Proof For an arbitrary semifinite trace, we have on the set $\left\{\rho \in \mathfrak{S}:\left\|h_{\rho}\right\|_{\infty} \leqslant c\right\}$

$$
\left|\tau\left(h_{\rho}^{\alpha}\right)^{\frac{1}{\alpha}}-\tau\left(h_{\varphi}^{\alpha}\right)^{\frac{1}{\alpha}}\right| \leqslant\left((2 c)^{\alpha-1}\left\|h_{\rho}-h_{\varphi}\right\|_{1}\right)^{\frac{1}{\alpha}}
$$

so if $\left\|\rho_{n}-\rho\right\|=\left\|h_{\rho_{n}}-h_{\rho}\right\|_{1} \rightarrow 0$, then $\tau\left(h_{\rho_{n}}^{\alpha}\right) \rightarrow \tau\left(h_{\rho}^{\alpha}\right)$, and thus $S_{\alpha}\left(\rho_{n}\right) \rightarrow S_{\alpha}(\rho)$, which proves the continuity of the Rényi entropy on the set $\left\{\rho \in \mathfrak{S}:\left\|h_{\rho}\right\|_{\infty} \leqslant c\right\}$ in the general case.

In the case $\mathcal{M}=\mathbb{B}(\mathcal{H})$ and the canonical trace, we have for every $h \in \mathcal{M}^{+},\|h\|_{\infty}=$ $\max \{\lambda: \lambda \in s p h\} \leqslant \operatorname{tr} h=\|h\|_{1}$, and thus

$$
\left\|h_{\rho}-h_{\varphi}\right\|_{\infty}^{\alpha-1} \leqslant\left(\left\|h_{\rho}\right\|_{\infty}+\left\|h_{\varphi}\right\|_{\infty}\right)^{\alpha-1} \leqslant\left(\left\|h_{\rho}\right\|_{1}+\left\|h_{\varphi}\right\|_{1}\right)^{\alpha-1} .
$$

Consequently, if $\left\|\rho_{n}-\rho\right\|=\left\|h_{\rho_{n}}-h_{\rho}\right\|_{1} \rightarrow 0$, then for the density matrices we have $\left\|h_{\rho_{n}}\right\|_{1} \leqslant c$, and hence $\left\|h_{\rho}\right\|_{1} \leqslant c$ for some $c$, so the estimate (10) holds, proving, as before, the continuity of the Rényi entropy on $\mathfrak{S}$.

The results obtained so far lead to the following theorem on semicontinuity of Segal's entropy.

Theorem 9 Segal's entropy is norm-upper semicontinuous on the set $\left\{\rho \in \mathfrak{S}:\left\|h_{\rho}\right\|_{\infty} \leqslant c\right\}$ for every $c \geqslant 0$. In the case $\mathcal{M}=\mathbb{B}(\mathcal{H})$ and the canonical trace, Segal's entropy is norm-upper semicontinuous on $\mathfrak{S}$ (consequently, von Neumann's entropy is norm-lower semicontinuous on $\mathfrak{S}$ ).

Proof For Segal's entropy H we have

$$
H(\rho)=\lim _{\alpha \rightarrow 1+}\left(-S_{\alpha}(\rho)\right),
$$

and Rényi's entropies $S_{\alpha}$ are continuous as functions of state on the set $\left\{\rho \in \mathfrak{S}:\left\|h_{\rho}\right\|_{\infty} \leqslant\right.$ $c$ ) for an arbitrary algebra $\mathcal{M}$, and the set $\mathfrak{S}$ for the algebra $\mathbb{B}(\mathcal{H})$, respectively. Since the net $\left\{-S_{\alpha}: \alpha>1\right\}$ is decreasing as $\alpha \rightarrow 1+, H$ is upper semicontinuous as a limit of a decreasing net of continuous functions. (Since von Neumann's entropy is minus Segal's entropy the lower semicontinuity of von Neumann's entropy follows.)

Remark 4 Lower semicontinuity of von Neumann's entropy was proved in [11], while the result as above for Segal's entropy was obtained in [6, Proposition II.7.6] by using a technique of rearrangements. A simple proof above unifies these two results.

Our last result concerns continuity of Segal's entropy in finite von Neumann algebras for not necessarily normalised states.

Theorem 10 Let $\mathcal{M}$ be a finite von Neumann algebra with a normal finite faithful trace $\tau$. Then Segal's entropy is norm-continuous on the set $\left\{\rho \in \mathcal{M}_{*}^{+}:\left\|h_{\rho}\right\|_{\infty} \leqslant c\right\}$ for every $c \geqslant 0$. 
Proof Let $\left\|\rho_{n}-\rho\right\|=\left\|h_{\rho_{n}}-h_{\rho}\right\|_{1} \rightarrow 0$. By virtue of Lemma $1, h_{\rho_{n}} \rightarrow h_{\rho}$ in measure. Let $f$ be the function defined as

$$
f(t)=t \log t, \quad t \in[0,+\infty) .
$$

Since $f$ is continuous, we get, on account of [7, Theorem 2.1],

$$
f\left(h_{\rho_{n}}\right) \rightarrow f\left(h_{\rho}\right) \text { in measure. }
$$

Since for the operators $h_{\rho_{n}}$ we have $s p h_{\rho_{n}} \subset[0, c]$, it follows that

$$
-\frac{1}{e} \mathbb{1} \leqslant f\left(h_{\rho_{n}}\right) \leqslant m \mathbb{1}
$$

for some $\mathrm{m}$, and consequently, there is a real number $\mathrm{M}$ such that

$$
-M \mathbb{1} \leqslant f\left(h_{\rho_{n}}\right) \leqslant M \mathbb{1} .
$$

By virtue of [9, Theorem 4.8] (see also [2, Theorem 3.6]), we infer that

$$
\left\|f\left(h_{\rho_{n}}\right)-f\left(h_{\rho}\right)\right\|_{1} \rightarrow 0,
$$

and the inequality

$$
\left|\tau\left(f\left(h_{\rho_{n}}\right)-f\left(h_{\rho}\right)\right)\right| \leqslant \tau\left(\mid f\left(h_{\rho_{n}}-f\left(h_{\rho}\right) \mid\right)=\left\|f\left(h_{\rho_{n}}\right)-f\left(h_{\rho}\right)\right\|_{1}\right.
$$

yields

$$
H\left(\rho_{n}\right)=\tau\left(f\left(h_{\rho_{n}}\right)\right) \rightarrow \tau\left(f\left(h_{\rho}\right)\right)=H(\rho),
$$

which finishes the proof.

Open Access This article is distributed under the terms of the Creative Commons Attribution 4.0 International License (http://creativecommons.org/licenses/by/4.0/), which permits unrestricted use, distribution, and reproduction in any medium, provided you give appropriate credit to the original author(s) and the source, provide a link to the Creative Commons license, and indicate if changes were made.

\section{References}

1. Bratteli, O., Robinson, D.W.: Operator Algebras and Quantum Statistical Mechanics, vol. 1. SpringerVerlag, New York-Heidelberg-Berlin (1987)

2. Fack, T., Kosaki, H.: Generalized s-numbers of $\tau$-measurable operators. Pac. J. Math. 123, 269-300 (1986)

3. Łuczak, A.: Laws of large numbers in von Neumann algebras and related results. Stud. Math. 81, 231243 (1985)

4. Nelson, E.: Notes on non-commutative integration. J. Funct. Anal. 15, 103-116 (1974)

5. Neshveyev, S., Strmer, E.: Dynamical Entropy in Operator Algebras. Springer, Berlin-Heidelberg-New York (2006)

6. Ohya, M., Petz, D.: Quantum Entropy and Its Use. Springer, Berlin-Heidelberg-New York (2004)

7. Padmanabhan, A.R.: Probabilistic aspects of von Neumann algebras. J. Funct. Anal. 31, 139-149 (1979)

8. Segal, I.E.: A note on the concept of entropy. J. Math. Mech. 9(4), 623-629 (1960)

9. Stinespring, W.F.: Integration theorems for gages and duality for unimodular groups. Trans. Am. Math. Soc. 90, 15-56 (1959)

10. Takesaki, M.: Theory of Operator Algebras II Encyclopaedia of Mathematical Sciences, vol. 125. Springer, Berlin-Heidelberg-New York (2003)

11. Wehrl, A.: Three theorems about entropy and convergence of density matrices. Rep. Math Phys. 10(2), 159-163 (1976)

12. Yeadon, F.J.: Non-commutative $l_{p}$-spaces. Math. Proc. Cambridge Philos. Soc. 77, 91-102 (1975) 